\title{
Investigation of Stress of Contact Wire Clamped with Steady Arms Under a Moving Constant Force Passing by
}

\author{
Takeo MORIKAWA \\ Senior Researcher, Contact Line Structure G., \\ Power Supply Technology Div.
}

\begin{abstract}
Presently, the maximum speed of Shinkansen trains reaches $300 \mathrm{~km} / \mathrm{h}$. In this highspeed operation, one of the factors in estimating the quality of current collecting behavior is the stress of contact wire. There are various fittings clamped to the contact wire of overhead catenary equipment. These fittings dynamically become hard spots for pantograph collecting current. Above all, large magnitudes of contact wire stress have been marked at steady arm points. Though being under statics, in order to reveal the fundamental properties in the relation between constrain conditions of steady arms and a moving constant force, analytic calculations are carried out.
\end{abstract}

Keywords : current collection, overhead line, contact wire, stress

\section{Introduction}

There are a number of fittings connected with the contact wire, e.g. steady arms, push-off arms, feeding branches and droppers. In the general section of the Shinkansen, the largest contact wire stress is normally marked at the point of steady arm. And this is one of the major causes of train speed restriction. In Japanese railways, it is estimated that the limit of the magnitude of contact wire stress is $60 \mathrm{MPa}$, which corresponds to 500 $\mu$ in strain. This takes the fatigue limit of contact wire materials into account. From this viewpoint, contact wires may allow 10 million passages of pantograph without breaking. Presently, it is understood from experiments that the characteristic of contact wire stress against the train velocity is in approximate accordance with the calculation results by the model of an infinite length tensioned beam on a stiffness bed ${ }^{1)}$. Therefore, it is desired to lower the stress of contact wire, which becomes larger as the train speed increases. Thus, this report describes the calculation results of contact wire stress under various steady arm conditions by pantograph passage.

\section{Calculation conditions}

\subsection{Calculation model and arrangement}

Fig. 1 indicates the calculation model. The contact wire is represented by a finite length tensioned beam clamped at both ends with a steady arm at its center. The deflection profile or stress distribution of the beam and time-dependence configuration of displacement or stress at arbitrary instances or points are examined while a constant uplift force moves from left end to right. Stress is calculated only on the opposite side of the sliding surface. Here, some assumptions are adopted the beam subjects to Bernoulli-Euler beam, tangential displacement of the beam is zero and tension along the beam is of constant magnitude and so on. In this paper, the following nomenclature is used.
T: the tension of beam, EI: rigidity of beam, $\rho$ :weight of unit length of beam, g: acceleration of gravity, $\mathrm{E}$ : Young's modulus, I: geometrical moment of inertia, Z: section modulus, $l$ : a half-length of a beam, $x$ : horizontal axis, $y$ : vertical axis, and $\mathrm{P}$ : moving force.

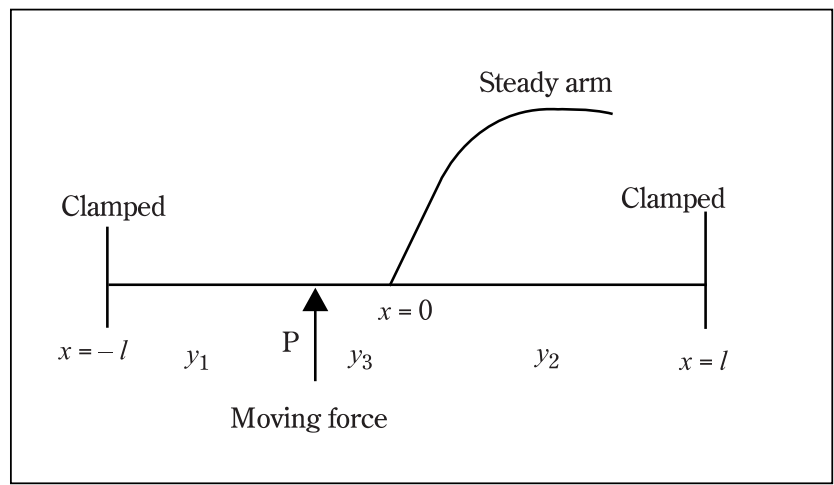

Fig. 1 Model arrangement for calculation

\subsection{Fundamental equations}

When deflection variables in each section are adopted as indicated in Fig. 1, their general solutions are given as follows.

$$
\begin{aligned}
& y_{1}=-\frac{\rho g}{2 T} x^{2}+C_{1} x+D_{1}+A_{1} e^{-\alpha x}+B_{1} e^{\alpha x} \cdots(1) \\
& y_{2}=-\frac{\rho g}{2 T} x^{2}+C_{2} x+D_{2}+A_{2} e^{-\alpha x}+B_{2} e^{\alpha x} \cdots(2) \\
& y_{3}=-\frac{\rho g}{2 T} x^{2}+C_{3} x+D_{3}+A_{3} e^{-\alpha x}+B_{3} e^{\alpha x} \cdots(3)
\end{aligned}
$$

where $\alpha=\sqrt{T /(E I)}$.

Fig. 2 indicates seven cases investigated on the various occasions of steady arm. Provided that boundary conditions at both ends are clamped, then the above equations become 
- Cond.1 No fitting

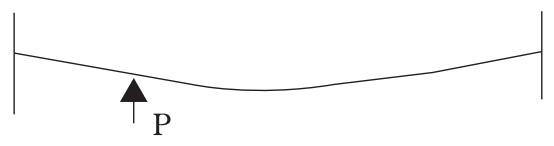

- Cond.2 Hinged junction, no vertical force

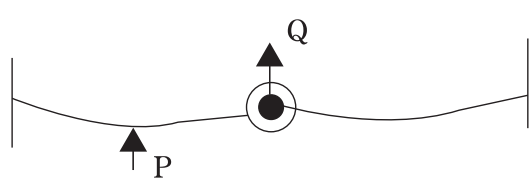

- Cond.3 Roller support

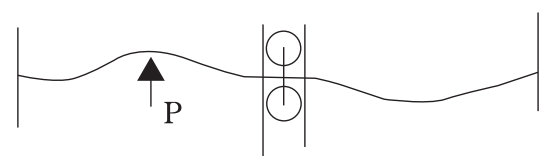

- Cond.4 Pinned support, vertical force acting on

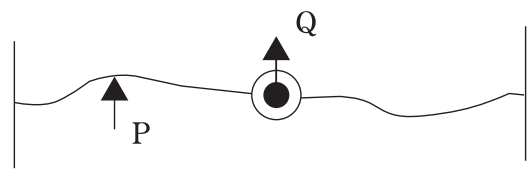

- Cond.5 Clamped support, fixed at a height

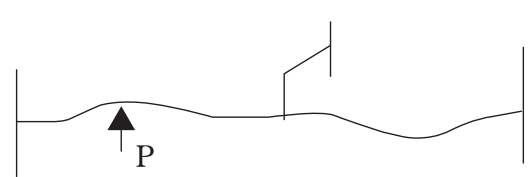

- Cond.6 Pinned support fixed at a height

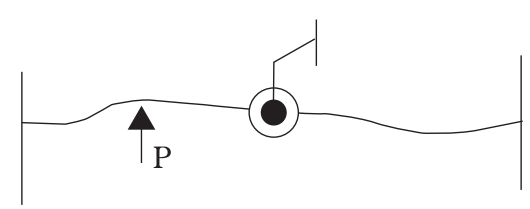

$$
\begin{gathered}
\left.y_{3}\right|_{x=0}=\left.y_{2}\right|_{x=0}, \\
\left.\frac{\partial y_{3}}{\partial x}\right|_{x=0}=\left.\frac{\partial y_{2}}{\partial x}\right|_{x=0}, \\
\left.\frac{\partial^{2} y_{3}}{\partial x^{2}}\right|_{x=0}=\left.\frac{\partial^{2} y_{2}}{\partial x^{2}}\right|_{x=0},\left.\frac{\partial^{3} y_{3}}{\partial x^{3}}\right|_{x=0}=\left.\frac{\partial^{3} y_{2}}{\partial x^{3}}\right|_{x=0},
\end{gathered}
$$

$$
\left.y_{3}\right|_{x=0}=\left.y_{2}\right|_{x=0},
$$$$
\left.T \frac{\partial y_{2}}{\partial x}\right|_{x=0}-\left.T \frac{\partial y_{3}}{\partial x}\right|_{x=0}+\left.\left(-E I \frac{\partial^{3} y_{2}}{\partial x^{3}}\right)\right|_{x=0} \text {, }
$$$$
-\left.\left(-E I \frac{\partial^{3} y_{3}}{\partial x^{3}}\right)\right|_{x=0}=Q \text {, }
$$$$
\left.\frac{\partial^{2} y_{3}}{\partial x^{2}}\right|_{x=0}=0,\left.\frac{\partial^{2} y_{2}}{\partial x^{2}}\right|_{x=0}=0
$$

$$
\begin{gathered}
\left.y_{3}\right|_{x=0}=\left.y_{2}\right|_{x=0}, \\
\left.\left(-E I \frac{\partial^{3} y_{2}}{\partial x^{3}}\right)\right|_{x=0}-\left.\left(-E I \frac{\partial^{3} y_{3}}{\partial x^{3}}\right)\right|_{x=0}=Q, \\
\left.\frac{\partial y_{3}}{\partial x}\right|_{x=0}=0,\left.\quad \frac{\partial y_{2}}{\partial x}\right|_{x=0}=0,
\end{gathered}
$$

$$
\begin{gathered}
\left.y_{3}\right|_{x=0}=\left.y_{2}\right|_{x=0}, \\
\left.\frac{\partial y_{3}}{\partial x}\right|_{x=0}=\left.\frac{\partial y_{2}}{\partial x}\right|_{x=0}, \\
\left.\left(-E I \frac{\partial^{3} y_{2}}{\partial x^{3}}\right)\right|_{x=0}-\left.\left(-E I \frac{\partial^{3} y_{3}}{\partial x^{3}}\right)\right|_{x=0}=Q, \\
\left.\frac{\partial^{2} y_{3}}{\partial x^{2}}\right|_{x=0}=\left.\frac{\partial^{2} y_{2}}{\partial x^{2}}\right|_{x=0}
\end{gathered}
$$

$$
\begin{aligned}
& \left.y_{3}\right|_{x=0}=y_{0},\left.\quad y_{2}\right|_{x=0}=y_{0}, \\
& \left.\frac{\partial y_{3}}{\partial x}\right|_{x=0}=0,\left.\quad \frac{\partial y_{2}}{\partial x}\right|_{x=0}=0
\end{aligned}
$$

$$
\begin{aligned}
& \left.y_{3}\right|_{x=0}=y_{0},\left.\quad y_{2}\right|_{x=0}=y_{0}, \\
& \left.\frac{\partial y_{3}}{\partial x}\right|_{x=0}=\left.\frac{\partial y_{2}}{\partial x}\right|_{x=0}, \\
& \left.\frac{\partial^{2} y_{3}}{\partial x^{2}}\right|_{x=0}=\left.\frac{\partial^{2} y_{2}}{\partial x^{2}}\right|_{x=0}
\end{aligned}
$$

$$
\begin{gathered}
\left.y_{3}\right|_{x=0}=0,\left.\quad y_{2}\right|_{x=0}=0 \\
\left.\frac{\partial y_{3}}{\partial x}\right|_{x=0}=\left.\frac{\partial y_{2}}{\partial x}\right|_{x=0}, \\
\left.\left(-E I \frac{\partial^{2} y_{3}}{\partial x^{2}}\right)\right|_{x=0}-\left.\left(-E I \frac{\partial^{2} y_{2}}{\partial x^{2}}\right)\right|_{x=0}=R
\end{gathered}
$$

Fig. 2 Arranged conditions of steady arm and their boundary conditions 


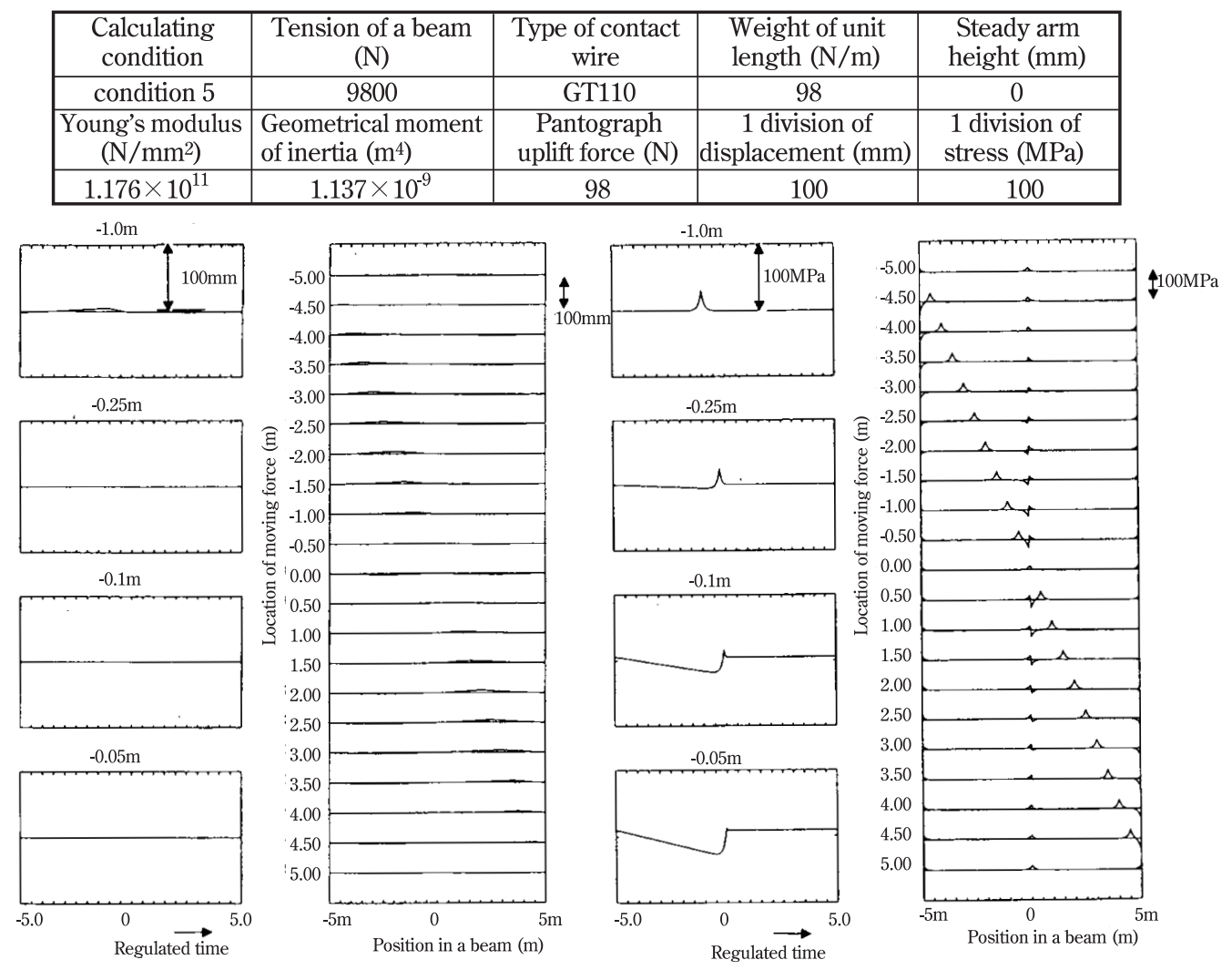

(a) Cond. 5: clamped support, steady arm fixed height 0mm

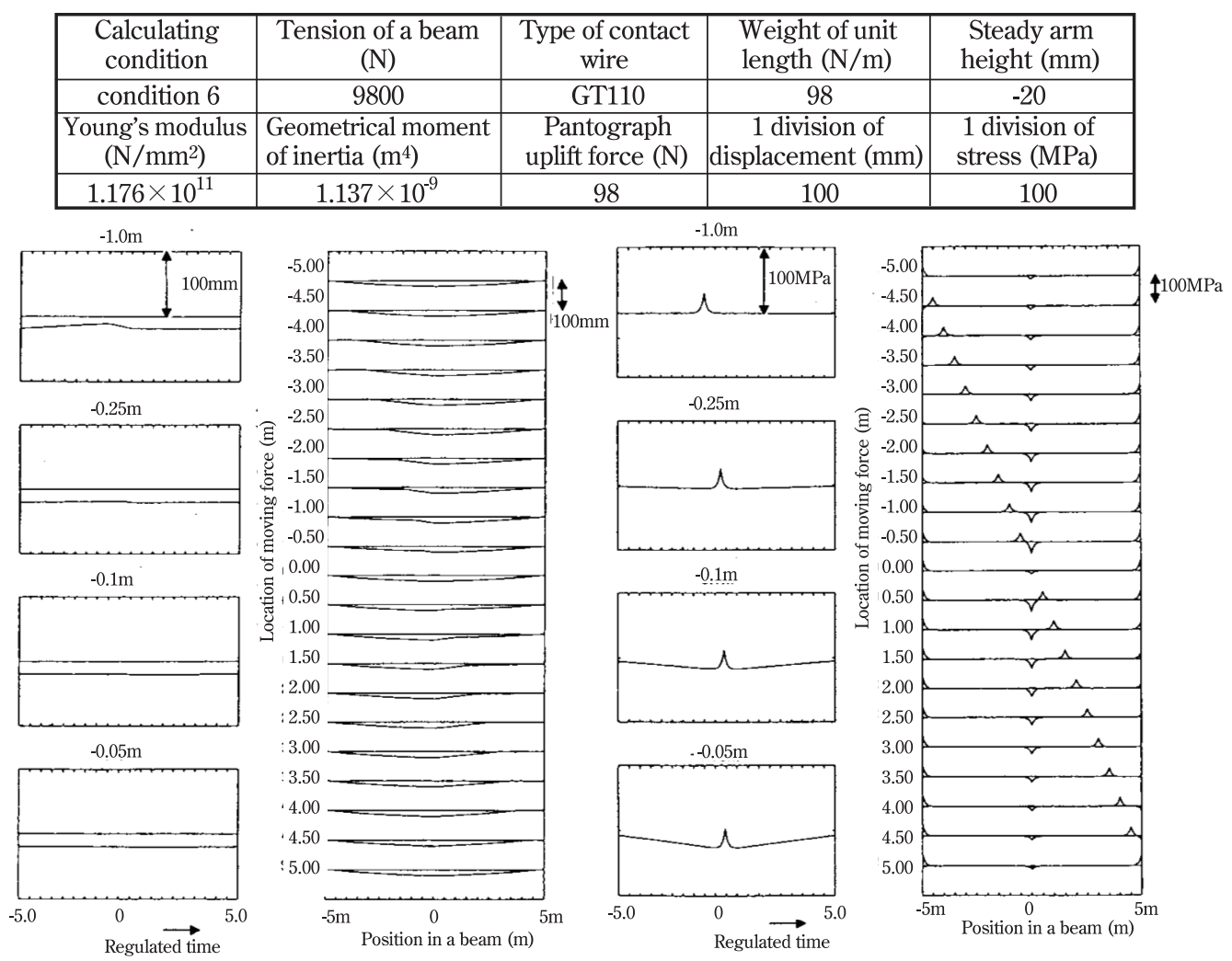

(b) Cond. 6: pinned support, steady arm fixed $-20 \mathrm{~mm}$

Fig. 3 Uplift displacement and stress configuration of a beam under a moving force 


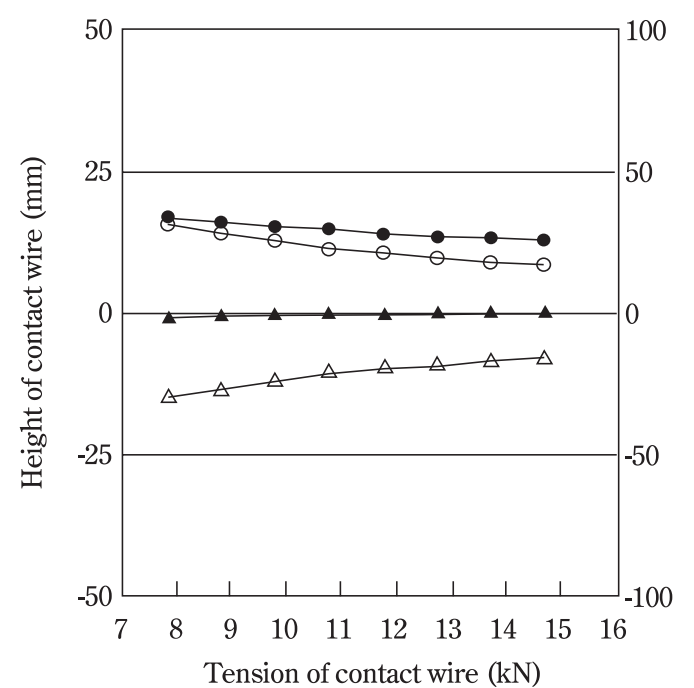

(a) Effect of tension (cond. 1, no fitting)

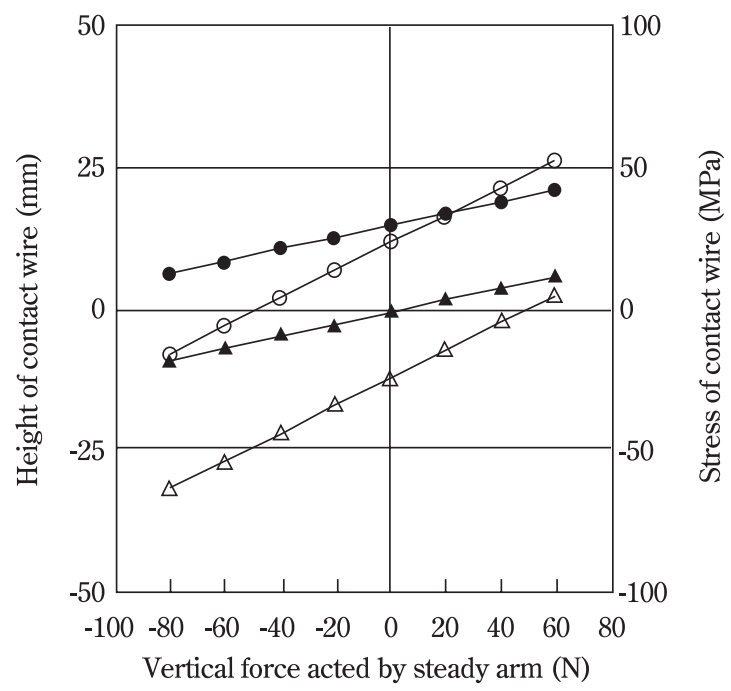

(c) Effect of vertical force (cond. 4, pinned support)

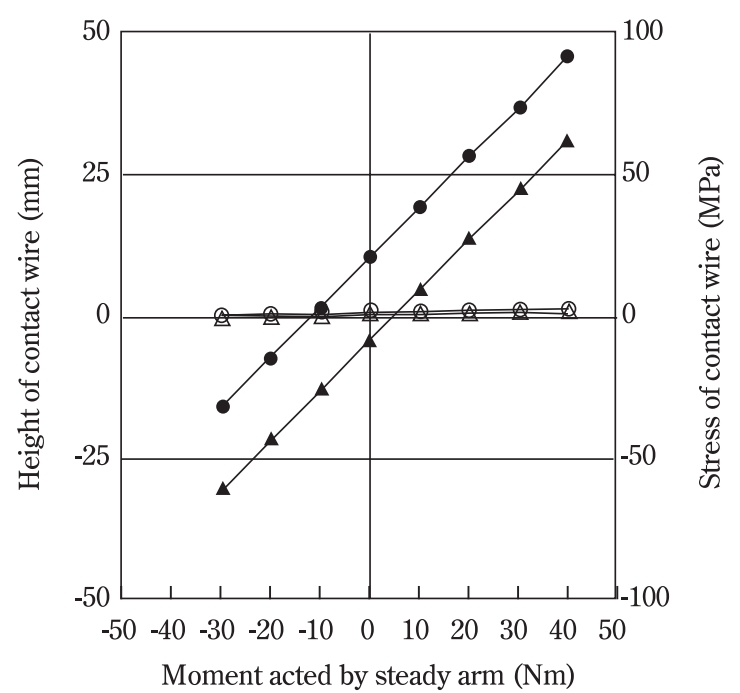

(e) Effect of moment (cond. 7, pinned support)

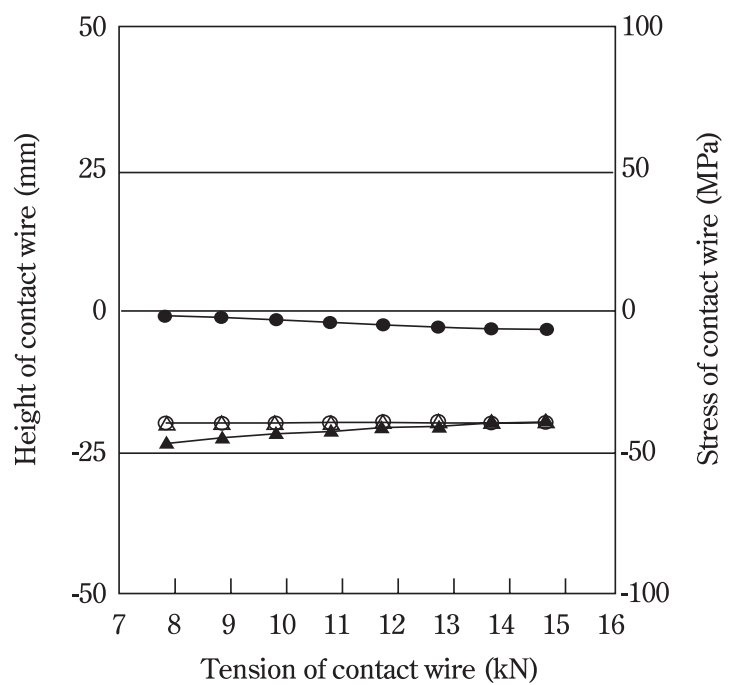

(b) Effect of tension (cond. 5, clampled support)

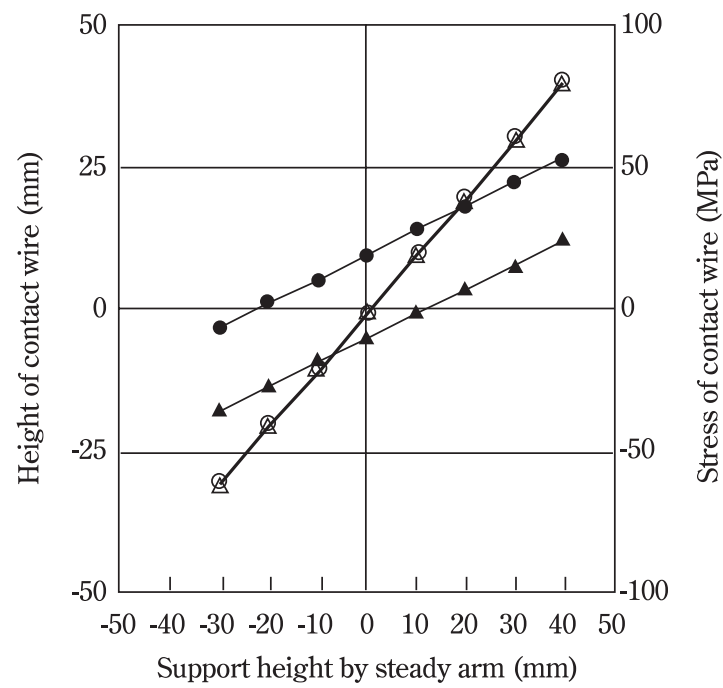

(d) Effect of support height (cond. 6, pinned support)

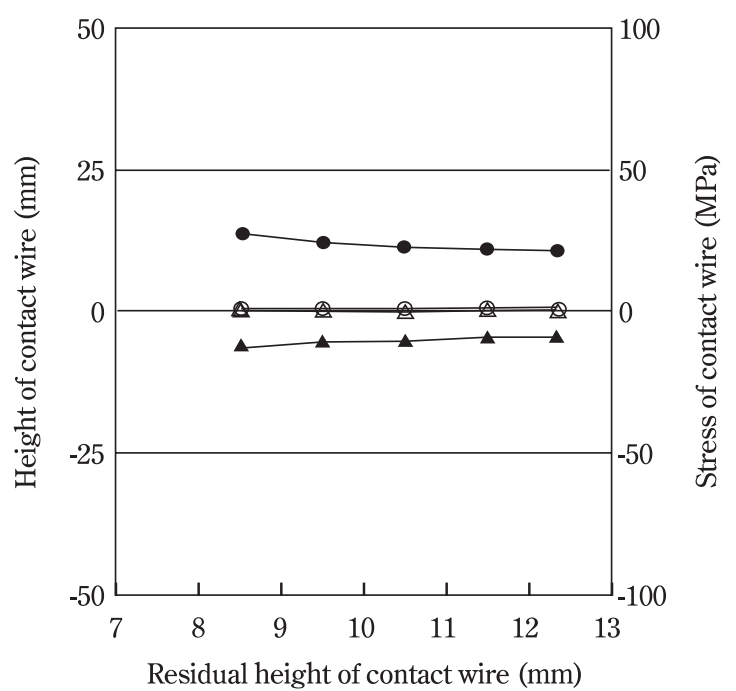

(f) Effect of residual height (cond. 7, pinned support)

$\bigcirc$ Maximum height, $\triangle$ Minimum height,

- Maximum stress

$\Delta$ Minimum stress

Fig. 4 Effect of some factors on stress under various steady arm conditions 


$$
\begin{gathered}
\left.y_{1}\right|_{x=-l}=0 \\
\left.\frac{\partial y_{1}}{\partial x}\right|_{x=-l}=0 \\
\left.y_{2}\right|_{x=l}=0 \\
\left.\frac{\partial y_{2}}{\partial x}\right|_{x=l}=0
\end{gathered}
$$

On the other hand, Fig. 2 also indicates the four boundary conditions at the point of steady arm located at the center of the beam. In addition, at a moving force point $\left(x=-x_{0}\right)$ there exist four boundary conditions as follows.

$$
\begin{aligned}
& \left.y_{1}\right|_{x=-x_{0}}=\left.y_{3}\right|_{x=-x_{0}} \\
& \left.\frac{\partial y_{1}}{\partial x}\right|_{x=-x_{0}}=\left.\frac{\partial y_{3}}{\partial x}\right|_{x=-x_{0}} \\
& \left.\frac{\partial^{2} y_{1}}{\partial x^{2}}\right|_{x=-x_{0}}=\left.\frac{\partial^{2} y_{3}}{\partial x^{2}}\right|_{x=-x_{0}} \\
& \left.\left(-E I \frac{\partial^{3} y_{3}}{\partial x^{3}}\right)\right|_{x=-x_{0}}-\left.\left(-E I \frac{\partial^{3} y_{1}}{\partial x^{3}}\right)\right|_{x=-x_{0}}=P
\end{aligned}
$$

Now that twelve boundary conditions are given, these equations can be solved.

\section{Results of calculation}

Fig. 3(a) and 3(b) show the results of calculation under cond.5 and 6, respectively. As for the stress at the steady arm, under cond.5 (clamped), its configuration resembles a 'breakwater', and also a 'hollow with a thorn at its center'. Fig. 5 indicates the typical stress configurations under various steady arm conditions. According to these configurations, they can be classified into three types, the above mentioned two and one with no fittings (cond.1). Fig. 4(a) shows the maximum and minimum heights of contact wire versus the tension of that in the case of no fittings, and its figure also shows the maximum and minimum stresses. The maximum and minimum heights of contact wire gradually decrease in magnitude as the tension of contact wire increases. On the other hand, while the maximum stress decreases as the tension of contact wire increases, the minimum stress is kept at zero in spite of the increase in tension. The latter means the reflection of uplift displacement property. Fig. 4(b) shows the same calculation under the condition that the steady arm is clamped at $-20 \mathrm{~mm}$ in height. Therefore, the steady arm rests at a constant height of $-20 \mathrm{~mm}$. On the other hand, the stress under this condition shows only minus values due to the configuration type of ' breakwater '. Fig. 4(c) shows the effect of vertical force in the cond.4. In this figure, the differences between the maximum and minimum values hold constant in spite of the variation of vertical force acted on the contact wire given by the steady arm. This means that the principle of superposition is effective between vertical and moving forces.
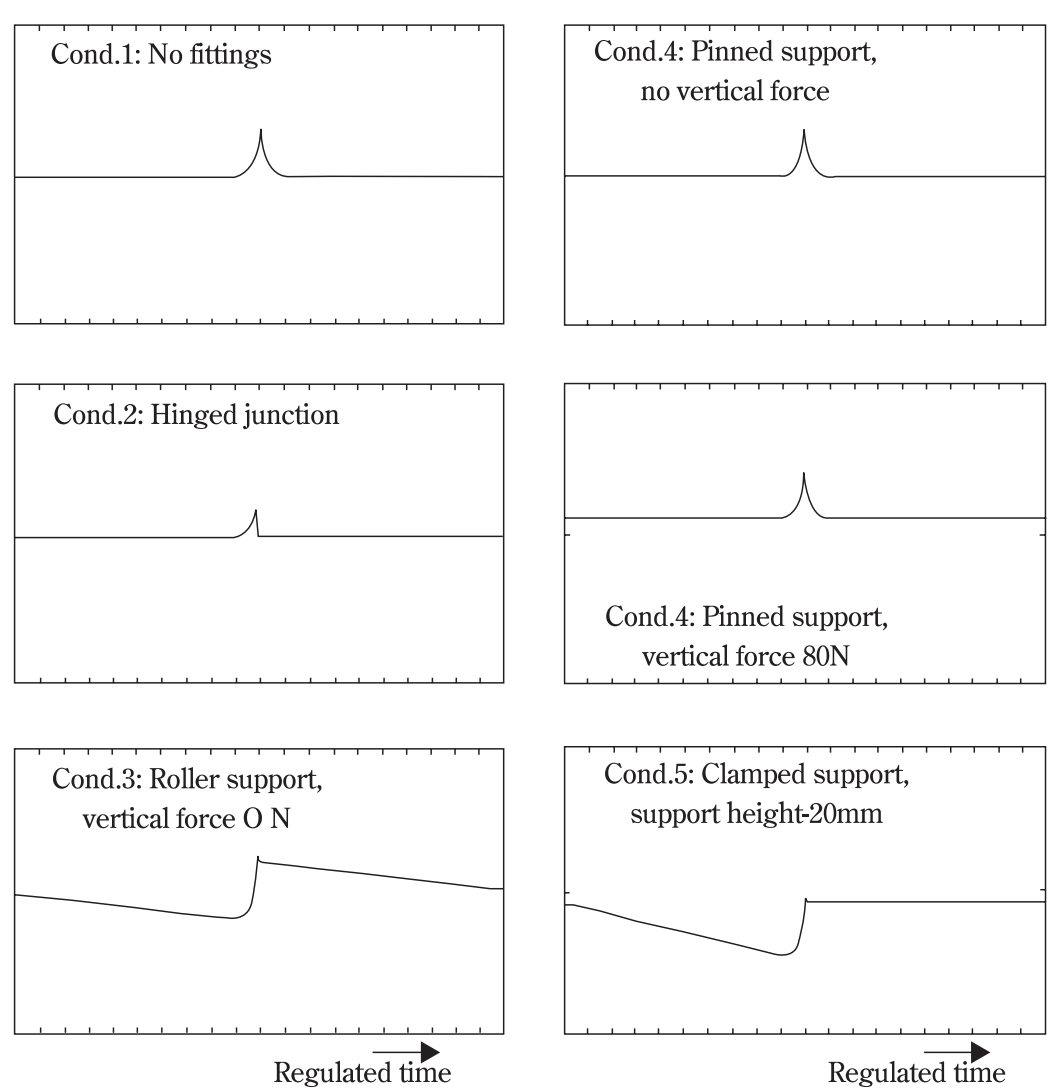

Cond.4: Pinned support vertical force $80 \mathrm{~N}$
Fig. 5 Typical stress configures by pantograph passages under various support (steady arm) conditions
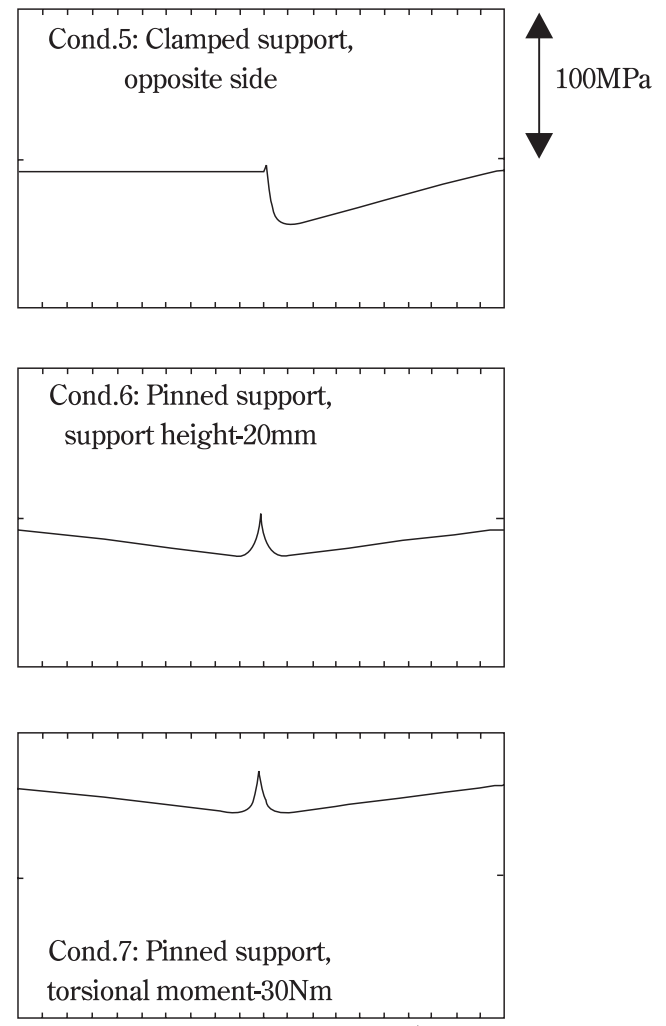

Regulated time 
Fig. 4(d) shows the effect of fixed height on stress. So, the height of steady arm accords with the preliminarily assigned value. Fig. 4(e) shows the effect of torsional moment acting on the contact wire by the steady arm. In this case, the contact wire at the steady arm is fixed at zero height. Fig. 4(f) shows the effect of residual diameter of contact wire on stress. Naturally, the stress increases as the residual diameter decreases.

Fundamentally the principle of superposition is valid under any steady arm condition, no matter what height the steady arm is fixed at or what magnitude of vertical force or torsional moment acted on.

\section{Conclusions}

Under various constrained conditions of steady arm, the following conclusions are gained for the contact wire stress produced by an uplift force passage.

(1) The stress configurations of contact wire at a steady arm are classified into three types under various conditions.Three representative configurations are ob- served under the condition of no fittings, clamped support and pinned support.

(2) As for the boundary conditions, the principle of superposition applies. All boundary conditions at a steady arm can be divided into two conditions. One is the zero value of $\mathrm{Q}, \mathrm{R}$ or $y_{0}$ and the other is some finite value. The sum of the solutions of the two which correspond to each boundary condition satisfies the original boundary conditions.

(3) In the case of no fittings, the maximum stress decreases as the tension of contact wire increases. On the other hand, the minimum stress remains at zero value as it does.

\section{References}

1) RTRI: "Property of Overhead Line and Pantograph", Ken-yusha, Japan, 1993.10

2) Manabe: “Improvement of Shinkansen's Overhead Catenary to High-Speed Operation", RTRI REPORT, Vol.4, No.12, 1990.12 